\title{
Produção científica acerca da Política Nacional de Práticas Integrativas e Complementares
}

\author{
Scientific production on the National Policy of Integrative and Complementary Practices \\ Producción científica sobre la Política Nacional de Prácticas Integrativas y \\ Complementarias
}

Isabela do Nascimento Souza ${ }^{1}$, Gislanne Batista Silva ${ }^{2}$, Karla Talita Santos Silva ${ }^{1}$, Marlete Scremin ${ }^{3}$, Cristiano Leonardo de Oliveira Dias ${ }^{4}$, Sylmara Corrêa Monteiro ${ }^{5}$, Bruna Gleide Mascarenhas Pinto ${ }^{6}$, Jéssica Najara Aguiar de Oliveira7 ${ }^{7}$, Ricardo Otávio Maia Gusmão ${ }^{4}$, Rene Ferreira da Silva Junior ${ }^{8 *}$.

\section{RESUMO}

Objetivo: Avaliar a produção científica associada à Política Nacional das Práticas Integrativas e Complementares após doze anos da sua implantação no Sistema Único de Saúde. Métodos: Estudo cienciométrico desenvolvido durante o primeiro semestre de 2019, utilizando o termo "Práticas Integrativas e Complementares", os artigos foram selecionados por meio de títulos, depois de selecionados os filtros de busca foram encontrados 417 publicações, sendo 50 na base de dados Scielo, 115 na Lilacs e 252 na Biblioteca Virtual em Saúde. Após avaliação de títulos e resumos foram excluídos artigos duplicados e que não fizessem referência a política, resultando em 37 artigos. Os dados foram tabulados e organizados em planilhas do programa Microsoft Excel 2010. Resultados: Não houve publicações no ano de 2006, que foi o ano da implantação da política, os estudos começaram a serem publicados a partir do ano de 2007, ocorreram maiores publicações no ano de 2012 e 2016, das modalidades das Práticas Integrativas e Complementares, as mais relatadas foram a fitoterapia, a acupuntura e a homeopatia, 15 das 29 práticas integrativas complementares, não foram abordadas nos estudos. Considerações finais: A produção científica acerca da Política Nacional de Práticas Integrativas e Complementares é escassa, considerando sua importância para a saúde pública.

Palavras-chave: Práticas integrativas e complementares, Políticas públicas, Sistema único de saúde.

\section{ABSTRACT}

Objective: To evaluate the scientific production associated with the National Policy of Integrative and Complementary Practices after twelve years of its implementation in the Unified Health System. Methods: Cienciometric study developed during the first half of 2019, using the term "Integrative and Complementary Practices", the articles were selected by means of titles, after selecting the search filters, 417 were found publications, 50 in the Scielo database, 115 at Lilacs and 252 in the Virtual Health Library. After evaluating titles and abstracts duplicate articles were excluded and not referencing the policy, resulting in 37 articles. The data was tabulated and organized into spreadsheets in the Microsoft Excel 2010 program. Results: There were no publications in 2006, which was the year of the implementation of the policy, the studies began to be

\footnotetext{
${ }^{1}$ Faculdades Unidas do Norte de Minas Gerais (FUNORTE), Montes Claros - MG.

*E-mail: renejunior deny@hotmail.com

${ }^{2}$ Centro Universitário Pitágoras (UniFipMoc), Montes Claros - MG.

3Instituto Federal de Educação, Ciência e Tecnologia (IFSC), Joinville - SC.

${ }^{4}$ Universidade Estadual de Montes Claros (Unimontes), Montes Claros - MG.

${ }^{5}$ Instituto Federal de Educação, Ciência e Tecnologia do Norte de Minas Gerais (IFNMG), Montes Claros MG.

6Universidade Federal de Minas Gerais (UFMG), Belo Horizonte - MG.

${ }^{7}$ Faculdade Santo Agostinho (FASA), Montes Claros - MG.

8Instituto Federal de Educação, Ciência e Tecnologia de Santa Catarina (IFSC), Joinville - MG.
} 
published from 2007, there were no major publications in 2012 and 2016, in the modalities of Integrative and Complementary Practices, the most reported were phytotherapy, acupuncture and homeopathy, 15 of the 29 complementary integrative practices, were not addressed in the studies. Final considerations: Scientific production on the National Policy of Integrative and Complementary Practices is scarce, considering its importance for public health.

Keywords: Integrative and complementary practices, Public policies, Unified health system.

\section{RESUMEN}

Objetivo: Evaluar la producción científica asociada a la Política Nacional de Prácticas Integrativas y Complementarias después de doce años de su implementación en el Sistema Unificado de Salud. Métodos: Estudio Cienciométrico desarrollado durante el primer semestre de 2019, utilizando el término "Prácticas Integrativas y Complementarias", los artículos fueron seleccionados por medio de títulos, después de seleccionar los filtros de búsqueda, se encontraron 417 publicaciones, 50 en la base de datos Scielo, 115 en Lila clas y 252 en la Biblioteca Virtual de Salud. Después de evaluar títulos y resúmenes, se excluyeron artículos duplicados y no hacían referencia a la política, lo que dio como resultado 37 artículos. Los datos se tabularon y organizaron en hojas de cálculo en el programa Microsoft Excel 2010. Resultados: No hubo publicaciones en 2006, que fue el año de la aplicación de la política, los estudios comenzaron a publicarse a partir de 2007, no hubo publicaciones importantes en 2012 y 2016, en las modalidades de Prácticas Integrativas y Complementarias, los más reportados fueron la fitoterapia, la acupuntura y la homeopatía, 15 de las 29 prácticas integrativas complementarias, no se abordaron en los estudios. Consideraciones finales: La producción científica sobre la Política Nacional de Prácticas Integrativas y Complementarias es escasa, considerando su importancia para la salud pública.

Palabras clave: Prácticas integrativas y complementarias, Políticas públicas, Sistema unificado de salud.

\section{INTRODUÇÃO}

As Práticas Integrativas e Complementares foram aprovadas no Sistema Único de Saúde (SUS) em três de maio de 2006 por meio da portaria gabinete do Ministro/ Ministério da Saúde (GM/MS) no 971 . A Política Nacional de Práticas Integrativas e Complementares (PNPIC), abrangia diretrizes e responsabilidades organizacionais para ofertar 6 serviços: homeopatia, medicina tradicional chinesa/acupuntura, plantas medicinais e fitoterapia, medicina antroposófica e termalismo social/crenoterapia (BRASIL, 2006).

Essa portaria foi alterada em março de 2017, a partir da Portaria Gabinete do Ministro no 849/2017, sendo contempladas à Política outras 14 prática: arteterapia, ayurveda, biodança, dança circular, meditação, musicoterapia, naturopatia, osteopatia, quiropraxia, reflexoterapia, reiki, shantala, terapia comunitária integrativa e yoga (BRASIL, 2018).

Já em 12 de março de 2018, durante a abertura do $1^{\circ}$ Congresso Internacional de Práticas Integrativas e Saúde Pública, no Rio de Janeiro, foi anunciada a inserção de 10 novas Práticas Integrativas e Complementares (PICS), sendo estas: apiterapia, aromaterapia, bioenergética, constelação familiar, cromoterapia, geoterapia, hipnoterapia, imposição de mãos, ozonioterapia e terapia de florais. Com as novas práticas, o Sistema Único de Saúde (SUS) passa a ofertar 29 procedimentos à população (BRASIL, 2018).

O Ministério da Saúde (MS) desenvolveu a Política de Práticas Integrativas e Complementares (PNPIC) tendo como objetivo ampliar a oferta de serviços oferecidos pelo Sistema Único de Saúde, proporcionando aos usuários, serviços de qualidade que anteriormente eram restritos a setores privados (NASCIMENTO MVN e OLIVEIRAIF, 2016). As PNPIC's baseiam-se no princípio de que a saúde é composta e desenvolvida por meio das pessoas por suas vivencias de cada dia. É constituída por cada um, pelo seu individual, e pelo coletivo, pela capacidade de tomar decisões e de ter controle sobre sua própria vida (MAGALHÃES MGM e ALVIM NAT, 2013). 
As PIC's se tornaram um dispositivo que tenciona algumas relações no campos da saúde pública, pois gera uma problematização em torno da garantia da integralidade numa perspectiva emancipadora, participativa e criativa, que articula saberes, práticas, vivências e espaços de saúde, contudo, apresenta uma contribuição para desmedicalização nos serviços de saúde (NASCIMENTO MVN e OLIVEIRA IF, 2016).

Assim, tais práticas se voltam à necessidade do ser humano acometido por um problema, ou um conjunto de manifestações clínicas que requerem cuidados para reestabelecer o equilíbrio de seu bem-estar físico, mental, social, espiritual, e outros capazes de atender as suas expectativas de saúde (MAGALHÃES MGM e ALVIM NAT, 2013).

As PIC's ainda são um modelo de possibilidades terapêuticas pouco acessadas, apesar da sua amplitude e eficácia, e é este o maior desafio da sua implantação. Entretanto, este modelo trouxe consideráveis avanços para a saúde, podendo ser destacado a capacidade de intervir sob problemas derivados de doenças já existentes (MELOS CC, et al., 2013).

Diante dessa realidade das Práticas Integrativas e Complementares no Brasil, objetivou-se avaliar o nível da produção cientifica associada à Política Nacional das Práticas Integrativas Complementares após doze anos da sua implantação no Sistema Único de Saúde.

\section{MÉTODOS}

Foi realizado estudo cienciométrico da literatura científica nacional sobre a Política Nacional de Práticas Integrativas Complementares (PNPICS), aprovada pelo Sistema Único de Saúde (SUS), em 3 de maio de 2006, por meio da Portaria GM/ MS no 971, e que vem sofrendo alterações ao longo dos seus 12 anos de implementação.

A pesquisa foi realizada nas seguintes bases de dados: SciELO, LILACS e BVS. Trata-se de um estudo baseado na cienciometria que é um ramo da pesquisa que se encarrega de avaliar a produção científica diante do número de publicações acerca de um assunto, sendo assim, este, o método escolhido para avaliar nível da produção cientifica associada à Política Nacional das Práticas Integrativas Complementares após doze anos da sua implantação no Sistema Único de Saúde.

A pesquisa foi realizada durante o primeiro semestre de 2019, utilizando o termo em português "Práticas Integrativas e Complementares". Foram critérios de inclusão: publicações de 2006 a 2018, artigos, estudo realizado com humanos e trabalhos na língua portuguesa, sendo inclusos 37 artigos. Como critérios de exclusão: artigos que não atendessem os critérios de inclusão, artigos duplicados, teses, dissertações, resumos e artigos em inglês, sendo excluídos 380 artigos, conforme fluxograma (Figura 1).

Os artigos foram selecionados inicialmente por meio de títulos que contivessem o termo "práticas alternativas e complementares", ou alguma variação deste e de títulos que contivessem a referência de alguma das 29 práticas abrangidas pela PNPICS. Em seguida, os artigos foram selecionados por meio da leitura dos resumos. Foram excluídos artigos que não tivessem como tema central a Política Nacional de Práticas Integrativas Complementares ou artigos que abordassem alguma prática alternativa que não fosse integrada a política, entre outros que não se enquadravam na temática do estudo.

Após leitura dos resumos, os trabalhos selecionados foram classificados, com o auxílio de instrumento elaborado pelos autores com os seguintes dados: ano de publicação; local de origem das publicações (baseado no primeiro autor); nome do periódico da publicação do trabalho; webqualis (A2, B1, B2, B3 e B4) na área interdisciplinar.

A classificação WebQualis refere-se à estratificação da qualidade da produção científica utilizada pela Coordenação de Aperfeiçoamento de Pessoal de Nível Superior (CAPES) para avaliar os periódicos nas diferentes áreas de pesquisa no Brasil. A seleção dos artigos foi realizada de forma independente pelos pesquisadores, as divergências foram resolvidas com o uso do instrumento elaborado. Após a seleção final foram extraídas as informações por meio do instrumento citado que guiou a elaboração das figuras e análise de dados. Os dados foram tabulados e organizados em planilhas do programa Microsoft Excel 2016. 
Figura 1 - Fluxograma do processo de estudo cienciométrico.

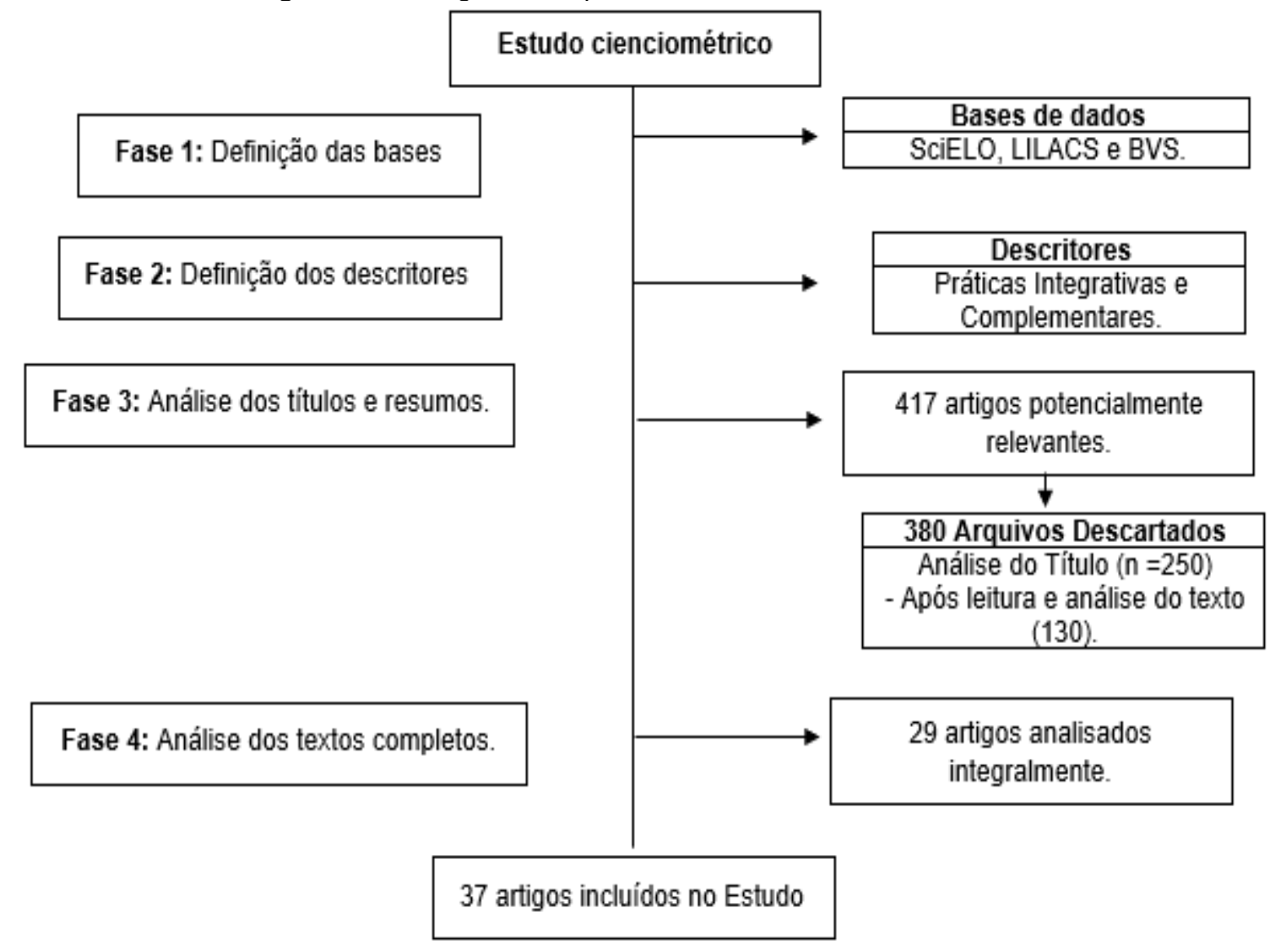

Fonte: Souza IN, et al., 2020.

\section{RESULTADOS}

$\mathrm{Na}$ etapa inicial, depois de selecionados os filtros de busca foram encontrados 417 publicações, sendo 50 na base de dados Scielo, 115 na Lilacs, e outras 252 na Biblioteca Virtual de Saúde (BVS). Após avaliação de títulos e resumos foram excluídos artigos duplicados, e artigos que não fizessem referência a PNPIC, resultando em uma amostra de 37 artigos.

Observa-se que nas bases de dados Scielo, Lilacs e BVS não houve publicações no ano de 2006, que foi o ano da implantação da Política (PNPIC), os estudos só começaram a serem publicados a partir do ano de 2007. Ocorreram maiores publicações no ano de 2012 e 2016 (Figura 2).

Considerando a PNPIC, e as Práticas Integrativas e Complementares, foi possível identificar que do total de 37 publicações, cerca de mais de 67, $60 \%$ (25) estão relacionados às Práticas Integrativas de uma forma geral, algumas vezes relacionando-se a alguma profissão ou doença específica, os outros $32,40 \%$ (12) estão relacionados a práticas específicas, sendo as mais prevalentes a acupuntura, a fitoterapia e a homeopatia, respectivamente.

Quanto à classificação dos artigos ao local de origem das pesquisas, nota-se que o estado de São Paulo $(27,02 \%)$, e Santa Catarina $(21,62 \%)$, são os estados brasileiros que detêm o maior número de publicações relacionadas à PNPIC. Dentre os outros estados que também apresentam publicações, figuram-se: Rio Grande do Norte, Pernambuco, Ceará, Goiás e Piauí (Figura 3).

Os resultados referentes ao qualis dos artigos analisados evidenciaram que a maioria das pesquisas foram publicadas em periódicos nos estratos qualis B1 e B2 (área interdisciplinar), representando 89,18 \% (33) (Figura 4). A Revista Ciência e Saúde Coletiva foi o periódico que apresentou o maior número de publicações acerca da PNPIC (Figura 5). 
Figura 2 - Número de artigos publicados nas bases de dados Scielo, Lilacs e BVS no período de 2006 a 2015 sobre a PNPIC.

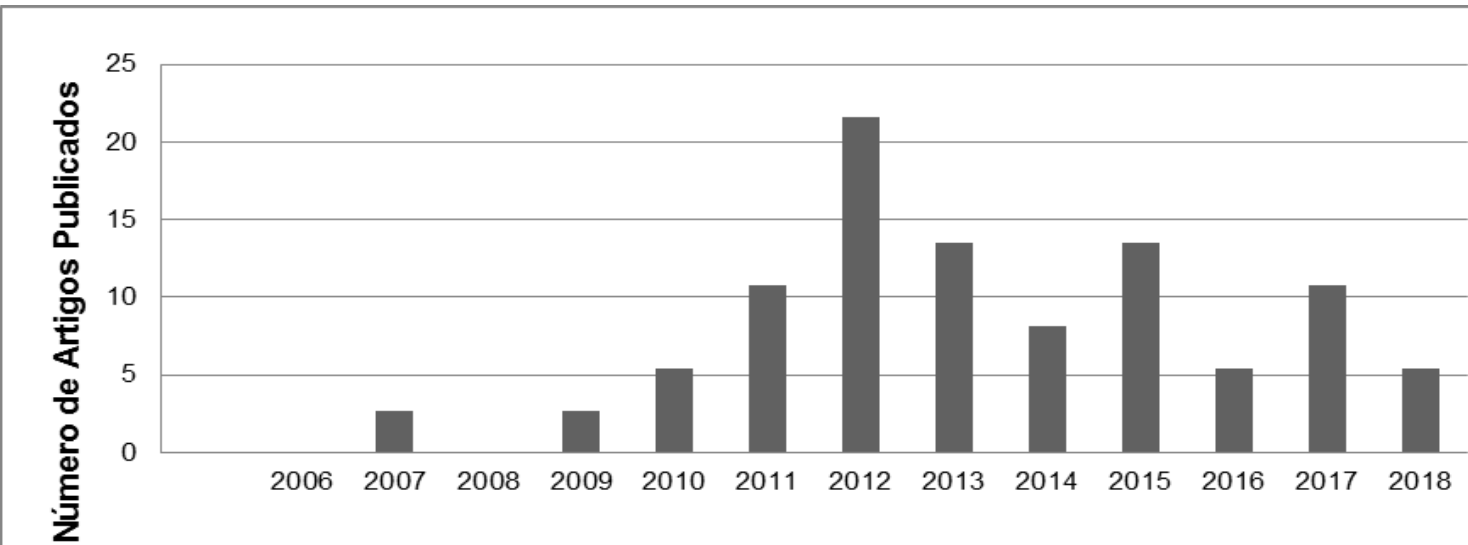

Ano da Publicação

Fonte: Souza IN, et al., 2020.

Figura 3 - Número de artigos publicados no período de 2006 a 2015 sobre a PNPIC, classificados quanto ao local de origem das publicações.

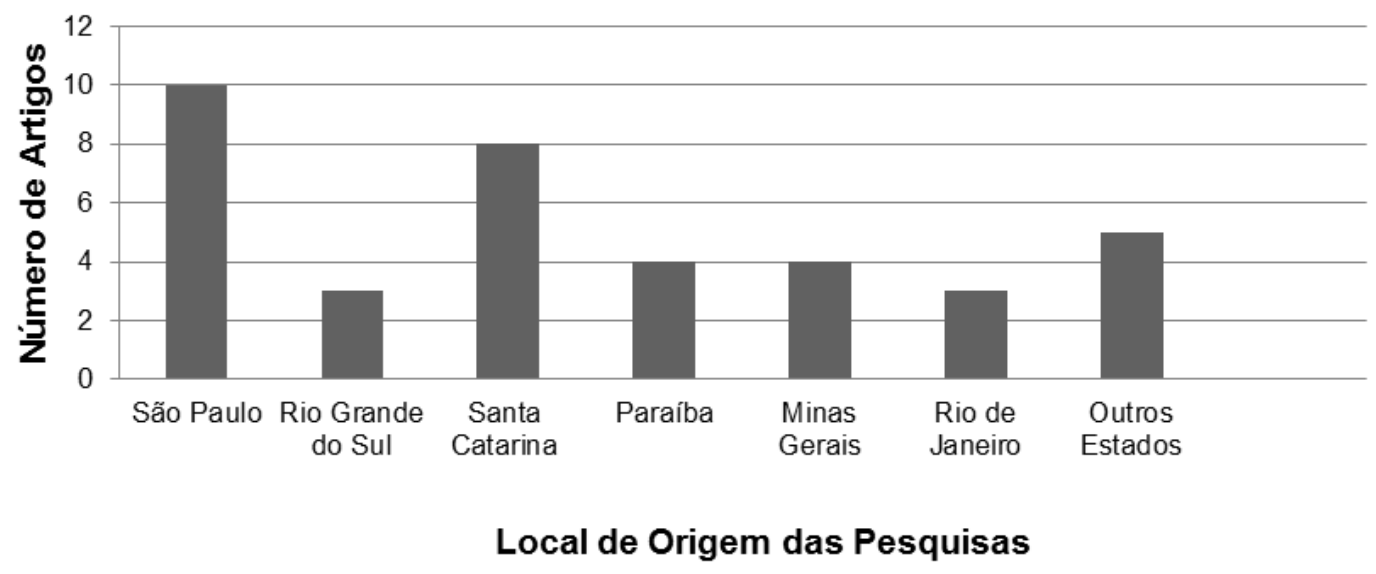

Fonte: Souza IN, et al., 2020.

Figura 4 - Levantamento do estrato Qualis/Capes dos periódicos científicos com publicações no período de 2006 a 2018 sobre a PNPIC.

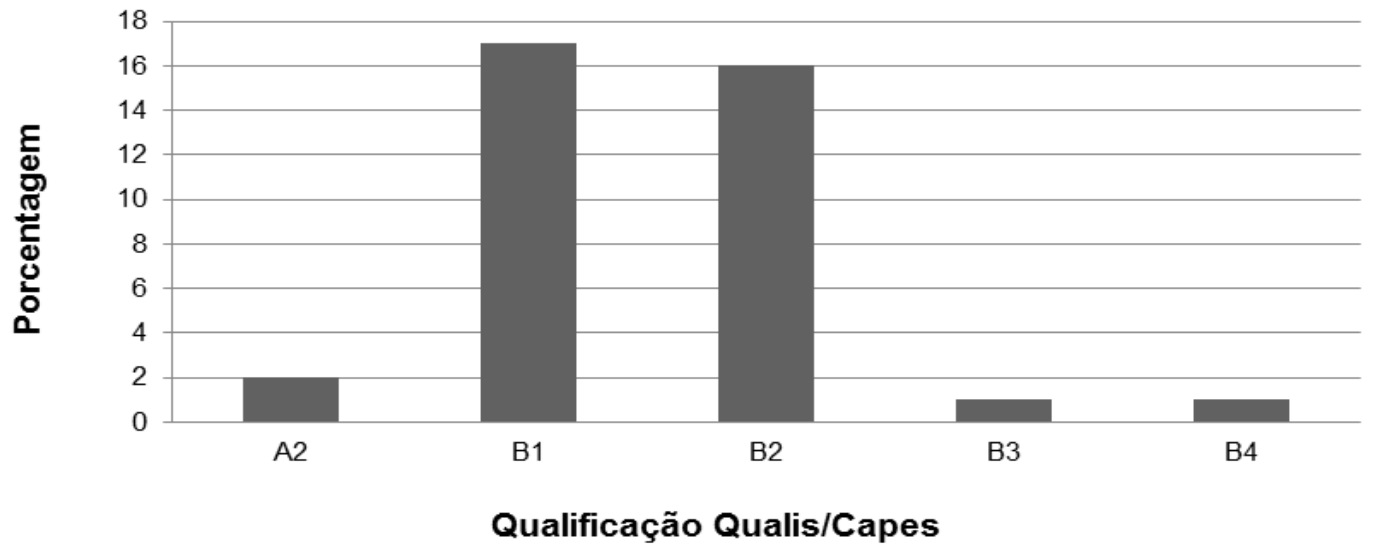

Fonte: Souza IN, et al., 2020. 
Figura 5 - Periódicos científicos com maior porcentagem de artigos publicados sobre a Política Nacional de Práticas Integrativas Complementares - PNPIC após implantação no ano de 2006 até 2018.

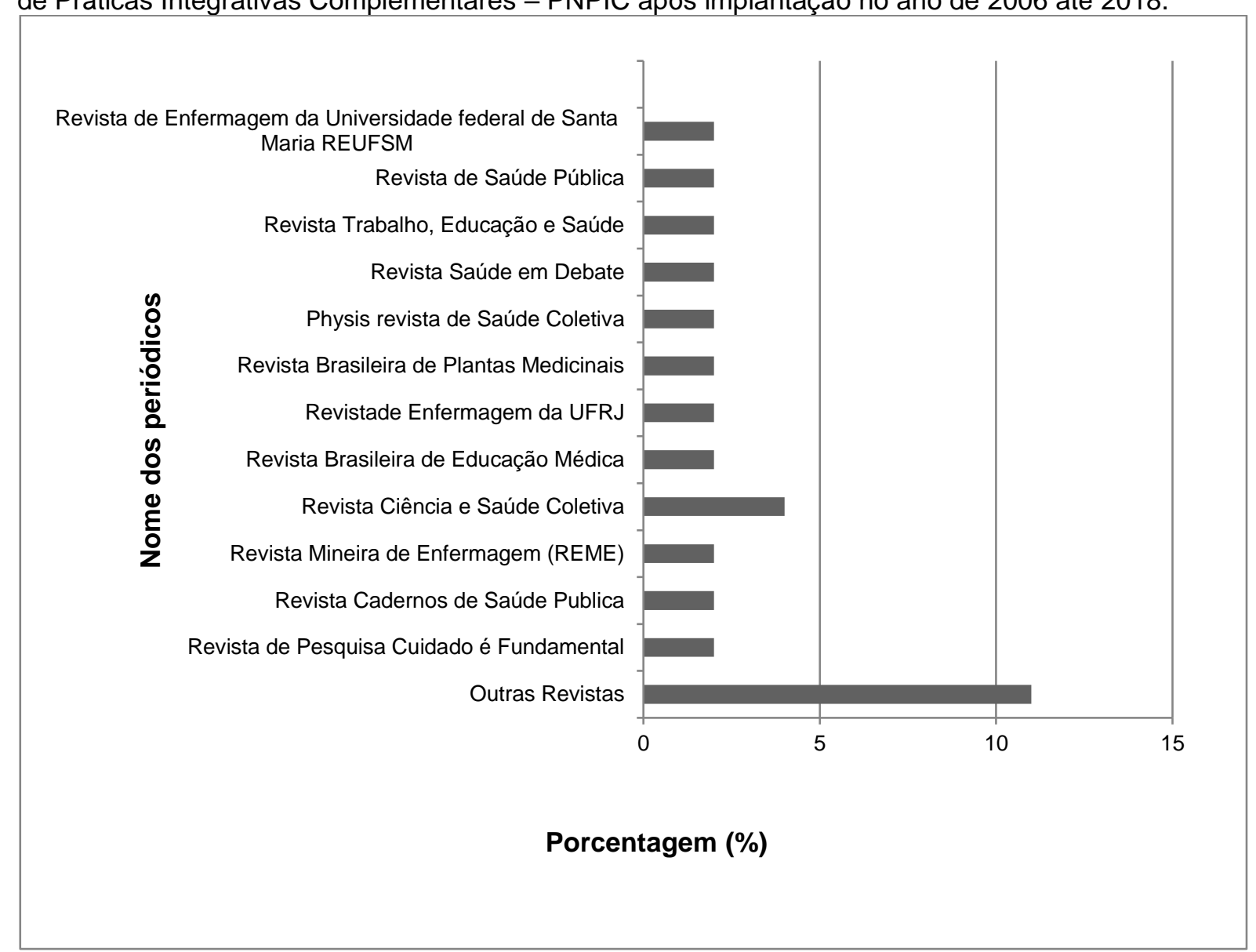

Fonte: Souza IN, et al., 2020.

\section{DISCUSSÃO}

No mundo inteiro surgem novas tecnologias a todo o momento, e na área da saúde não é diferente. Novos procedimentos e técnicas de curar são admitidos a qualquer custo, muitas vezes em detrimento a humanização. Tal humanização vem aos poucos sendo resgatada no cuidado com o paciente, interligando a união do corpo, mente e ambiente (SANTOS LF e CUNHA AZ, 2011).

Nesse contexto, a Política Nacional de Práticas Integrativas e Complementares surge propondo implantar seu serviço se baseando na humanização do cuidado, centrando na integralidade do indivíduo, além de atuar nas esferas de prevenção de agravos, promoção, manutenção e recuperação da saúde, como proposta de fortalecer os princípios do SUS (NASCIMENTO MVN e OLIVEIRA IF, 2016).

O efeito da inclusão da Política Nacional de Práticas Integrativas e Complementares no Sistema Único de Saúde atinge não só o âmbito da saúde, mas outros campos também, como o sociopolítico, econômico, técnico, o ensino e a pesquisa. O desenvolvimento das práticas deve amenizar os impactos sociais, físicos e psicológicos, devem ser incentivadas com o foco em promoção e melhoria da qualidade de vida (BESSA JHN e OLIVEIRA DC, 2013; SARAIVA AM, et al., 2015).

As práticas devem ser ofertadas com intuito de oferecer e promover um cuidado humanizado, visando melhorar a qualidade assistencial da saúde, sendo estas também vistas como possibilidades terapêuticas, pois, são recursos extremamente úteis na promoção da saúde no âmbito do SUS, pois estabelece uma diferente compreensão do processo saúde-doença, se destacando na perspectiva holística e individual, tornando-se impactante na vida do paciente (SCHVEITZER MCI, et al., 2012; MELO SCC, et al., 2013; LIMA KMSV, et al., 2014). 
Não foi em vão que o SUS implantou as práticas integrativas complementares, é perceptível a efetividade destas sobre o bem-estar e a melhoria da saúde dos usuários. No geral, as PICS são um modelo de ampliação da atenção à saúde, de forma integral. É preciso, considerar que a Política Nacional de Práticas Integrativas Complementares esbarra em alguns problemas para a sua execução, dentre eles o mais observado foi o desconhecimento dos profissionais acerca da PNPIC (TESSER, CD 2009; SANTOS MS, et al., 2018; SANTOS MC e TESSER CD, 2012; SOUZA MRM, et al., 2012).

Um aspecto importante para o funcionamento das PNPICS é que os profissionais de saúde tenham conhecimento acerca das práticas integrativas e complementares, para que estas sejam inseridas no processo do cuidar, dado não retratado nos estudos analisados, pois se observou desconhecimento e desinformação por parte destes profissionais, consequentemente há ausência de divulgação e programas sociais que busquem possibilitar o acesso às práticas integrativas pelos pacientes. Com isto se dá a importância da inserção das PICs na formação dos profissionais de saúde, o que fortaleceria a PNPIC e levaria reconhecimento, apoio e respeito às práticas, além de melhorar a oportunidade de acesso dos pacientes a estas práticas.

Ressalta-se que a educação permanente em saúde aos profissionais já inseridos na malha social do Sistema Único de Saúde pode atender a demanda e é também um fator de incentivo a produção da pesquisa científica sobre a temática (MACHADO DCS, et al., 2012; GONTIJO MBA e NUNES MF, 2017; CARVALHO JLS e NÓBREGA MPSS, 2017; AZEVEDO E PELICIONI MCF, 2012). As PIC's mostraram ter um maior acesso e resolubilidade na atenção básica. É onde as pessoas têm buscado cada vez mais alternativas de cuidados que atendam a todas as suas necessidades, sejam elas físicas, psicológicas ou espirituais.

A insatisfação com a medicina tradicional se dá principalmente pela falta de informação aos pacientes sobre o seu tratamento e cura, além de outras decepções com o meio de saúde moderno, que abrange listas de esperas e restrições financeiras, efeitos colaterais de medicamentos e tratamentos e intervenções cirúrgicas são fatores que tem levado essas pessoas a procurarem formas alternativas de tratamento. Os profissionais que estão inseridos nas práticas integrativas e complementares têm um maior vínculo com o universo cultural e psicossocial dos usuários (LOSSO LN e FREITAS SFT, 2017; SARAIVA AM, et al., 2011; OTANI MAP e BARROS NF, 2011; SOUSA IMC e TESSER CD, 2016).

A prevalência do uso de práticas integrativas se dá pela inclusão ou não das terapias no sistema de saúde de cada país. Elas têm sido usadas em vários âmbitos na saúde como, por exemplo, na atuação das doulas, trazendo o respeito e autonomia da mulher, propondo um novo modelo de humanização do parto, na osteoporose, na ansiedade, na hipertensão arterial, dentre outras (MANTOVANI MF, et al., 2016; SILVA RM, et al., 2015).

Dentre os trabalhos utilizados, a área de atuação na saúde que mais se figura nos estudos é a enfermagem. Com a demanda brasileira sobre as práticas integrativas e complementares, nota-se que 0 enfermeiro é um profissional fundamental no cuidado, com grande responsabilidade em ações diagnósticas e orientações preventivas e terapêuticas (THIAGO SCS e TESSER CD, 2011).

A enfermagem deve reivindicar a regulamentação do emprego de PICS pelo SUS, e reivindicar as possibilidades de legitimação destas práticas no âmbito do cuidado de enfermagem emancipando-se, de modo a ofertar um cuidado mais amplo, humano e capaz de potencializar a autonomia do outro. Nota-se há a necessidade dos profissionais de saúde em geral, discutirem sobre a sua prática nos diversos âmbitos de atuação, agindo em consonância com as políticas públicas (MAGALHÃES MGM e ALVIM NAT, 2013; PENNAFORT VPS, et al., 2012; RANGEL CT, et al., 2016).

Acerca das modalidades das PIC's, as mais relatadas foram a fitoterapia, a acupuntura e a homeopatia, na respectiva ordem. A humanidade sempre fez uso de elementos medicamentosos encontrados na natureza para curar seus males, esse uso evoluiu, mas não deixou de existir. A chamada fitoterapia, se mostrou uma das práticas integrativas e complementares mais utilizadas no âmbito do SUS, e seu uso apresentou um crescimento devido à aproximação da Medicina Tradicional Chinesa à Medicina Ocidental (FEITOSA MHA, et al., 2016; KUBA G e VATTIMO MFF, 2015). 
A fitoterapia é uma das práticas terapêuticas, mais antigas da humanidade (cerca de 8.500 a.C.), apresenta conhecimentos populares e científicos, denomidados respectivamente como etnobotânica e etnofarmacologia. As plantas possuem princípios ativos com poder curativo, sendo a partir deste conhecimento, o nascimento da medicina alopática moderna. O termo Fitoterapia deriva do grego therapeia (tratamento), e phyton (vegetal) e faz referência ao estudo das plantas medicinais e suas aplicações em casos doenças, com intuito curativista (UNIVERSIDADE FEDERAL DE JUIZ DE FORA; 2010).

Em 2006, após a criação da Política Nacional de Práticas Integrativas e Complementares no SUS (PNPIC), que além da fitoterapia, contempla mais quatro práticas, foi criada também, em 22 de junho a Política Nacional de Plantas Medicinais e Fitoterápicos, por meio de portaria do Ministério da Saúde GM/MS no 971 que contribuiu para o incremento do uso de plantas medicinais. As duas políticas trazem a discussão sobre implantação da Fitoterapia nos serviços públicos de saúde, levando em consideração a oportunidade, a importância, as dificuldades, as facilidades e as vantagens da prática (SOUZA MRM, et al., 2012; FIGUEREDO CA, et al., 2014).

É importante que a fitoterapia seja fortalecida por meio das políticas públicas de saúde, com a criação de estudos a partir da medicina tradicional com ênfase nas plantas medicinais de interesse do SUS. Além disso, têm sido criados programas e politicas que reforçam a importância de o profissional de saúde ter conhecimento na prescrição de fitoterápicos para evitar o uso indevido e errôneo pela população, podendo, inclusive, diminuir os casos de intoxicação (MARQUES MAA, et al., 2016; OLIVEIRA VB, et al., 2018).

Reforça-se que a terapia fitoterápica traz mudanças significativas na vida dos usuários, contribuindo para a melhoria da qualidade de vida, pois trata holisticamente o indivíduo, trazendo um bem estar anteriormente não experimentado o que mostra que o uso de fitoterápicos no nível primário de atenção esteja relacionado às próprias demandas apresentadas pela comunidade. Para o uso da fitoterapia é exigida comprovação científica do efeito das plantas e avaliação das possíveis interações com medicamentos, efeitos adversos e toxicidade, nesse contexto a PNPIC incentiva a pesquisa de cada prática integrativa e complementar visando à melhoria da atenção à saúde e a avaliação dos cuidados oferecidos (LOURES MC, et al., 2019; VARELA DSS e AZEVEDO DM, 2014; GELATTI GT, et al., 2015).

A acupuntura é o conjunto de conhecimentos técnico-empíricos da medicina tradicional chinesa que objetiva a terapia e a cura das doenças por meio da aplicação de agulhas e de moxas. Surgiu há aproximadamente 4.500 anos, e apesar da sua antiga origem, continua em constante evolução. Segundo a sua teoria, o nosso organismo encontra-se em equilíbrio pela atuação das energias yin (negativo) e yang (positivo), desde modo se houver um desequilíbrio, ocorrerão doenças. Sendo assim, a acupuntura visa estimular pontos reflexos que tenham a propriedade de reestabelecer o equilíbrio, alcançando-se assim, resultados terapêuticos (WEN TS, 2006).

Ainda sobre as modalidades das PIC's, atualmente, a acupuntura é exercida no Brasil por diversos profissionais da área da saúde, e vem sendo reconhecida pelo conselho de cada profissão, principalmente após a PNPIC. Há um estigma em torno da acupuntura, atualmente mais brando do que em outras gerações, estigma que foi atribuído ao desconhecimento dos benefícios terapêuticos da prática (LOSSO LN e LOPES SS, 2018; ROLAND MIF e GIANINI RJ, 2012).

A homeopatia é um meio de tratamento considerado holístico e natural. Holístico porque considera o ser humano como um todo, e natural porque os medicamentos são produzidos através de substâncias de origem animal, mineral e vegetal. Baseada na cura pelos semelhantes, ou seja, uma mesma substância que em grande quantidade pode ser prejudicial a uma pessoa sadia, pode curar um doente, se diluída em várias doses. Foi desenvolvida por Samuel Hahnemann no fim do século XVIII, que descobriu que à medida que as doses eram diluídas, se tornavam mais eficazes. A homeopatia é um dos principais meios de tratamentos de doenças no mundo, ocupando o segundo lugar, com mais de 200 milhões de usuários (ASSOCIAÇÃO MÉDICA HOMEOPATICA BRASILEIRA, 2020).

Discute-se que a homeopatia é uma prática que tem aplicação em todas as faixas etárias. A sua implantação como opção terapêutica no SUS, juntamente com a ampliação do acesso, vem reforçar os 
princípios de universalidade, integralidade e equidade. A homeopatia também é uma prática que se mostrou desconhecida por grande parte dos profissionais da saúde, apesar de ser uma das mais utilizadas, o que novamente traz à tona a necessidade de capacitação aos profissionais e estudos na área. Por fim, constatouse que pelo menos 15 das 29 práticas integrativas complementares, não foram abordadas nas pesquisas (LOCH-NECKE G, et al., 2010; GALHARDI WMP, et al., 2013).

\section{CONSIDERAÇÕES FINAIS}

Com o presente estudo conclui-se que há uma baixa produção científica acerca da Política Nacional de Práticas Integrativas e Complementares no decorrer dos seus doze anos de implantação. A análise cienciométrica identificou que a fitoterapia, a acupuntura e a homeopatia são as práticas mais abordadas nas pesquisas, e constatou-se que pelo menos 15 das 29 práticas integrativas complementares, não foram abordadas em nenhum dos artigos. Esta pesquisa restringiu-se ao termo Práticas Integrativas e Complementares e algumas variações desde, levando em consideração as pesquisas que abordavam a PNPIC, que regulamenta as práticas no Sistema Único de Saúde. Em relação atemática, sugerem-se minuciosas investigações futuras, que permitam evoluir no conhecimento em torno das Práticas Alternativas e Complementares para promoção, prevenção, recuperação e reabilitação da saúde dos usuários.

\section{REFERÊNCIAS}

1. ASSOCIAÇÃO MÉDICA HOMEOPÁTICA BRASILEIRA. História. A homeopatia no Brasil. 2020. Disponível em: https://amhb.org.br/amhb/. Acesso em 02/09/2020.

2. AZEVEDO E PELICIONI MCF. Práticas integrativas e complementares de desafios para a educação. Revista Trabalho, Educação e Saúde, 2012; 9(3): 361-378.

3. BESSA JHN e OLIVEIRA DC. O uso da terapia Reiki nas Américas do Norte e do Sul: uma Revisão.Revista Enfermagem UERJ, 2013; 21(1): 660-664.

4. BRASIL. Manual de implantação de serviços de práticas integrativas e complementares no SUS/Ministério da Saúde, Secretaria de Atenção à Saúde. Brasília, 2018.

5. BRASIL. MINISTÉRIO DA SAÚDE. Portaria ํㅜ 971, de 03 de maio de 2006. Aprova a Política Nacional de Práticas Integrativas e Complementares (PNPIC) no Sistema Único de Saúde.

6. CARVALHO JLS e NÓBREGA MPSS. Práticas integrativas e complementares como recurso de saúde mental na Atenção Básica. Revista Gaúcha de Enfermagem, 2017; 4(38): 1-9.

7. FEITOSA MHA. Inserção do Conteúdo Fitoterapia em Cursos da Área de Saúde. Revista Brasileira de Educação Médica, 2016; 40(2): 197-203.

8. FIGUEREDO CA, et al. A Política Nacional de Plantas Medicinais e Fitoterápicos: construção, perspectivas e desafios. Physis Revista de Saúde Coletiva, 2014; 24(2): 381-400.

9. GALHARDI WMP, et al. O conhecimento de gestores municipais de saúde sobre a Política Nacional de Prática Integrativa e Complementar e sua influência para a oferta de homeopatia no Sistema Único de Saúde local. Revista Ciência \& Saúde Coletiva, 2013; 18(1): 213-220.

10. GONTIJO MBA, NUNES MF. Práticas Integrativas E Complementares: Conhecimento e Credibilidade de Profissionais do Serviço Público de Saúde. Revista Trabalho, educação e Saúde, 2017; 15(1): 301-320.

11. KUBA G, VATTIMO MFF. O uso de fitoterápicos orientais nas lesões renais: revisão integrativa. Revista Brasileira de Plantas Medicinais, 2015; 17(4): 1192-1198.

12. LIMA KMSV, et al. Práticas integrativas e complementares e relação com promoção da saúde: experiência de um serviço municipal de saúde. Revista Interface-Comunicação, Saúde, Educação, 2014; 18(49): 261-272.

13. LOCH-NECKE LG, et al. A homeopatia no SUS na perspectiva de estudantes da área da saúde. Revista Brasileira de Educação Médica, 2009; 34(1): 82-90.

14. LOSSO LN e FREITAS SFT. Avaliação do grau da implantação das práticas integrativas e complementares na Atenção Básica em Santa Catarina, Brasil. Revista Saúde em Debate, 2017; 41(3): 171-187.

15. LOSSO LN e LOPES SS. Análise da oferta e produção de atendimento em acupuntura na atenção básica em Santa Catarina. Revista Tempus Actas em Saúde Coletiva, 2018; 11(2): 159-177.

16. LOURES MC, et al. Contribuições da Fitoterapia para a Qualidade de Vida: Percepções de seus Usuários. Revista Enfermagem da UERJ. Goiânia, 2009; 18(2): 278-283.

17. MACHADO DCS, et al. Percepções de coordenadores de unidades de saúde sobre a fitoterapia e outras práticas integrativas e complementares. Revista Saúde em Debate, 2012; 36(95): 615-623.

18. MAGALHÃES MGM e ALVIM NAT. Práticas Integrativas e Complementares no cuidado de Enfermagem: um enfoque ético. Revista Escola Anna Nery Revista de Enfermagem, 2013; 17(4): 646-653.

19. MANTOVANI MF, et al. Utilização de Terapias Complementares por pessoas com Hipertensão Arterial Sistêmica. Revista Baiana de Enfermagem, 2016; 30(4): 1-8. 
20. MARQUES MAA, et al. Caracterização das plantas medicinais e medicamentos fitoterápicos para tratamento da osteoporose utilizados no Brasil. Revista Arquivos de Ciências da Saúde da UNIPAR, 2016; 20(3): 183-188.

21. MELO SCC, et al. Práticas complementares de saúde e os desafios de sua aplicabilidade no hospital: visão de enfermeiros. Revista Brasileira de Enfermagem, 2013; 66(6): 840-846.

22. NASCIMENTO MVN e OLIVEIRA IF. As práticas integrativas e complementares grupais e sua inserção nos serviços de saúde da atenção básica. Revista Estudos de Psicologia, 2016; 21(3):272-281.

23. OLIVEIRA VB, et al. Conhecimento e Uso de Plantas Medicinais por Usuários de Unidades Básicas de Saúde na Região de Colombo, PR. Revista Brasileira de Ciências da Saúde, 2018; 22(1): 57-64.

24. OTANI MAP e BARROS NF. A Medicina Integrativa e a construção de um novo modelo na saúde. Revista Ciência \& Saúde Coletiva, 2008; 16(3):1801-1811.

25. PENNAFORT VPS, et al. Práticas Integrativas e o Empoderamento da Enfermagem. Revista Mineira de Enfermagem, 2012; 16(2): 289-295.

26. RANGEL CT, et al. A terapia comunitária integrativa e a enfermagem: o fenômeno e seus contextos. Revista de Pesquisa Cuidado é Fundamental Online, 2016; 8(1): 3770-3779.

27. ROLAND MIF e GIANINIRJ. Redes sociotécnicas de assistência à saúde em acupuntura: estudo de caso sobre a formação básica de estudantes de medicina. Revista História, Ciências, Saúde, 2014; 201(2): 477-511.

28. SANTOS MS, et al. Práticas Integrativas e Complementares: avanços e desafios para a promoção da saúde de idosos. Revista Mineira de Enfermagem, 2018; 22(1): 1-5.

29. SANTOS LF, CUNHA AZ. A utilização de Práticas Complementares por enfermeiros do Rio Grande do Sul. Revista de Enfermagem da Universidade Federal de Santa Maria, 2011;1(3):369-376.

30. SANTOS MC, TESSER CD. Um método para a implantação e promoção de acesso às Práticas Integrativas e Complementares na Atenção Primária à Saúde. Revista Ciência \& Saúde Coletiva, 2012; 17(11): 3011-3024.

31. SARAIVA AM et al. Histórias de cuidados entre idosos institucionalizados: as Práticas Integrativas como possibilidades terapêuticas. Revista de Enfermagem da Universidade Federal de Santa Maria, 2015; 5(1):131-140.

32. SARAIVA AM, FILHA MOF, DIAS MD. As práticas integrativas como forma de complementaridade ao modelo biomédico: concepções de cuidadoras. Revista de Pesquisa Cuidado é Fundamental Online, 2011; Ed.Supl: $155-163$.

33. SCHVEITZER MCI, ESPER MV, SILVA MJP. Práticas Integrativas e Complementares na Atenção Primária em Saúde: em busca da humanização do cuidado. Revista O Mundo da Saúde, 2012; 36(3): 442-451.

34. SILVA RM et al. Uso de práticas integrativas e complementares por doulas em maternidades de Fortaleza (CE) e Campinas (SP. Revista Saúde e Sociedade, 2015; 25(1): 108-120.

35. SOUSA IMC, TESSERCD. Medicina Tradicional e Complementar no Brasil: inserção no Sistema Único de Saúde e integração com a atenção primária. Cadernos de Saúde Pública, 2016; 33(1): 1-15.

36. SOUZAMRM et al. Comercialização de plantas medicinais no contexto da cadeia produtiva em Minas Gerais. Revista Brasileira de Plantas Medicinais, 2012; 14(1): 242-245.

37. TESSER CD. Práticas complementares, racionalidades médicas e promoção da saúde: contribuições poucos exploradas. Revista Cadernos de Saúde Pública, 2009; 25(8): 1732-1742.

38. THIAGO SCS, TESSER CD. Percepção de médicos e enfermeiros da Estratégia de Saúde da Família sobre terapias complementares. Revista de Saúde Pública, 2010; 45(2): 249-257.

39. UNIVERSIDADE FEDERAL DE JUIZ DE FORA. Programa de plantas medicinais e terapias não convencionais. Disponível https://www.uff.br/proplamed/atividades/fitoterapia/\#: :text=O\%20termo\%20Fitoterapia\%20deriva\%20do,independe ntemente\%20na\%20maioria\%20dos\%20povos. Acesso em: 02/07/2020.

40. VARELA DSS, AZEVEDO DM. Saberes e práticas fitoterápicas de médicos na estratégia saúde da família. Revista Trabalho, educação e Saúde, 2014; 12(2): 273-290.

41.WEN, TS. Acupuntura Clássica Chinesa - São Paulo: Cultrix, 2006 . Disponível em: https://books.google.com.br/books?hl=ptBR\&lr=\&id=lJLwg1qXtagC\&oi=fnd\&pg=PA9\&dq=ACUPUNTURA\&ots=uiDnF yXa6Z\&sig=1 m4gxcsHDaHw3dCY19OXZkL2nFk\#v=onepage\&q=ACUPUNTURA\&f=false. Acesso em: 02/07/2020. 